\title{
Sind Ökonomie und Gesundheit miteinander vereinbar?
}

\section{Hans Stalder}

Prof. Dr. med., Mitglied der Redaktion

\begin{tabular}{|l|}
\hline WAS MAN \\
FÜR GELD \\
NICHT \\
KAUFEN \\
KANN \\
Dienumatichon \\
MICHAELJ. \\
SANDEL
\end{tabular}

Literatur

1 Hartzband P, Groopman J. Money and the changing culture of medicine. NEJM. 2009;360:101-2.

2 Schweizerische Akademie der Medizinischen Wissenschaften. Medizin und Ökonomie - wie weiter? Schweizerische Ärztezeitung. 2014;95:1598-16oo. 3 Sandel MJ. Was man für Geld nicht kaufen kann. Berlin: Ullstein;2014.

4 Dengler-Voss C, Voss R. Was fordert der Patient von mir, dem Hausarzt, und welche Wünsche verbergen sich hinter diesen Forderungen? PrimaryCare. 2014;14:289-91.

Siehe zu diesem Thema auch den Artikel von Johannes Bircher auf Seite 283.

\author{
Assigning a monetary value to every aspect \\ of a physician's time and effort may \\ actually reduce productivity, impair ... \\ quality ... and thereby even increase costs [1].
}

Die Schweizerische Akademie der Medizinischen Wissenschaften hat unlängst ein Positionspapier mit dem Titel «Medizin und Ökonomie - wie weiter?» veröffentlicht, in dem auch eine Reihe von Empfehlungen abgegeben wurden [2]. Der Ruf nach Bewahrung der heutzutage immer mehr bedrohten Solidarität zwischen Gesunden und Kranken, Alten und Jungen, Männern und Frauen kann nur befürwortet werden. Schwieriger zu akzeptieren ist die Beteuerung, dass «Medizin und Ökonomie nicht als Systeme mit konkurrierenden Zielsetzungen betrachtet werden dürfen, sondern vielmehr als Konzept mit gemeinsamem Ziel». Ökonomie zielt auf eine effiziente Zuweisung der Ressourcen nach der Kosten-Nutzen-Analyse. In der Medizin hingegen lässt sich nicht alles in wirtschaftliche oder nutzenbasierte Leistung übersetzen. Verbesserung der Gesundheit oder eine Verringerung von Leiden sind quantitativ nur schwer zu erfassen. In seinem Buch «Was man für Geld nicht kaufen kann. Die moralischen Grenzen des Marktes» [3] zeigt der an der Havard-Universität lehrende Ökonomieprofessor Michael Sandel an Beispielen, dass gegenwärtig praktisch alles käuflich ist. In der Medizin können (in einigen Ländern) Arzttermine ohne Wartezeit, immer gleich für den nächstfolgenden Tag, Organe für die Transplantation oder Leihmütter gekauft werden ... Und doch gibt es Grenzen: Freundschaft beispielsweise ist nicht käuflich, (echte) Liebe auch nicht. In der Medizin stellt sich daher die Frage, was in Geld ausgedrückt werden kann und was nicht.

Ich nehme an, in einem Röntgeninstitut sollte das möglich sein: Die Arbeitszeit des Technikers und des Radiologien können erfasst werden, wie auch die Amortisierung der Geräte und der Mietzins der Räumlichkeiten. Nur die Höhe der Vergütung der Beteiligten könnte zu Kontroversen führen. In der Allgemeinmedizin sind die Probleme anders. Die in einer Schweizer Studie [4] - die viele andere bestätigt - beschriebenen Wünsche von Patienten an den Allge- meinmediziner (s. Tabelle) lassen sich nur teilweise geldwert erfassen. Vielleicht das Fachwissen, das man manchmal messen oder in Zahlen ausdrücken kann, indem man bei Durchsicht von Krankengeschichten kontrolliert, ob anerkannte Guidelines angewendet wurden. Wie aber soll man Freundlichkeit, Empathie oder Geduld in Geld erfassen?

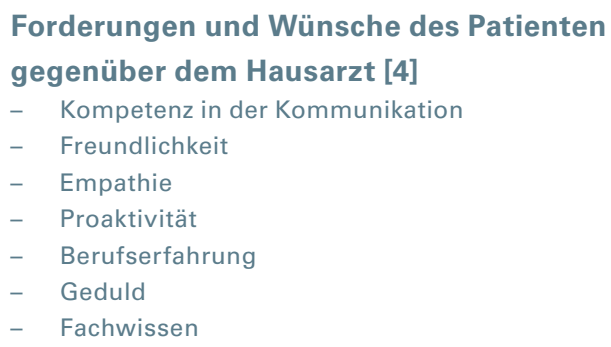

Dazu kommt, dass Geld Werte pervertieren kann. Dies lässt sich am Beispiel der Geschenke zeigen [3]. Fragt man den Empfänger nach dem Geldwert eines Geschenks, so wird dieser im Schnitt auf 80\% des realen Werts geschätzt. Um kein Fünftel auf unsere Geschenke zu verlieren, sollten wir aus rein ökonomischer Sicht nur Geldgeschenke machen. Geschenke haben jedoch nicht nur einen monetären Wert. Ihr «moralischer Wert» [3] kann ein freundschaftlicher Gedanke oder der Ausdruck von Liebe sein. Gibt der Verlobte seiner Geliebten anstelle des Verlobungsrings Geld, so wird die im Geschenk enthaltene Liebesbotschaft pervertiert. Wenn wir in der Medizin Werte wie Empathie, Geduld oder Freundlichkeit in geldwerten Einheiten ausdrücken wollen, werden sie korrumpiert. Dazu kommt, dass einmal kommerzialisierte und pervertierte moralische Haltungen kaum rückgängig gemacht werden können.

Aus der obengenannten Studie [4] wird deutlich, dass unsere Patienten diese nicht monetär auszudrückenden «moralischen Werte» in der Medizin sehr zu schätzen wissen. Gemeinsam mit unseren Patienten gilt es, diese Werte zu verteidigen und klar Nein zu sagen: Marktwirtschaft gilt nur für gewisse Leistungen in der Medizin, für andere jedoch nicht. Diese anderen Leistungen - sind's Geschenke? - lassen sich nicht beziffern. 